\title{
Bioanalysis
}

\section{White Paper on recent issues in bioanalysis: focus on new technologies and biomarkers (Part 1 - small molecules by LCMS)}

\begin{abstract}
The 2015 9th Workshop on Recent Issues in Bioanalysis (9th WRIB) took place in Miami, Florida with participation of over 600 professionals from pharmaceutical and biopharmaceutical companies, biotechnology companies, contract research organizations and regulatory agencies worldwide. It is once again a 5-day week long event - a full immersion bioanalytical week - specifically designed to facilitate sharing, reviewing, discussing and agreeing on approaches to address the most current issues of interest in bioanalysis. The topics covered included both small and large molecules, and involved LCMS, hybrid LBA/LCMS, LBA approaches including the focus on biomarkers and immunogenicity. This 2015 White Paper encompasses recommendations that emerged from the extensive discussions held during the workshop, and is aimed to provide the bioanalytical community with key information and practical solutions on topics and issues addressed, in an effort to advance scientific excellence, improve quality and deliver better regulatory compliance. Due to its length, the 2015 edition of this comprehensive White Paper has been divided into three parts. Part 1 covers the recommendations for small molecule bioanalysis using LCMS. Part 2 (hybrid LBA/LCMS and regulatory agencies' inputs) and Part 3 (large molecule bioanalysis using LBA, biomarkers and immunogenicity) will also be published in volume 7 of Bioanalysis, issues 23 and 24, respectively.
\end{abstract}

The 9th Workshop on Recent Issues in Bioanalysis (9th WRIB) was held in Miami, Florida on 13-17 April, 2015 with participation of over 600 professionals from pharmaceutical and biopharmaceutical companies, biotechnology companies, contract research organizations, and regulatory agencies worldwide. The workshop included three sequential core workshop days and six specialized training sessions that together spanned an entire week in order to allow exhaustive and thorough coverage of major issues in bioanalysis, biomarkers and immunogenicity. Like the previous workshops, the 9th WRIB was specifically designed to facilitate sharing, reviewing, discussing and agreeing on approaches to address the most current issues of interest in both small and large molecule bioanalysis using LCMS, hybrid LBA/LCMS and LBA approaches. An in depth focus was on biomarkers, immunogenicity and emerging technologies.
The actively contributing chairs in the 2015 edition of the WRIB were Dr E Fluhler (Pfizer), Dr J Welink (EMA/Dutch MEB), Dr B Ackermann (Eli Lilly), Dr F Garofolo (Angelini Pharma), Dr A Song (Genentech), Dr T Thway (Amgen), Dr L Amaravadi (Biogen Idec) and Dr H Myler (Bristol-Myers Squibb).

The numerous regulatory agency representatives who contributed to the 9th WRIB included Dr S Haidar (US FDA), Dr S Kirshner (US FDA), Dr B Booth (US FDA), Dr M Skelly (US FDA), Dr N Tampal (US FDA), Dr J Welink (EMA/Dutch MEB), Dr O Le Blaye (France ANSM), Ms E Whale (UK MHRA), Mr S Vinter (UK MHRA), Dr B Witte (German BfArM), Dr M Bustard (Health Canada), Mr G Mendes Lima Santos (Brazil ANVISA) and Dr N Katori (Japan MHLW-NIHS).

Each of the three sequential core workshop days was designed to cover a wide range of
Jan Welink', Eric Fluhler' Nicola Hughes ${ }^{3}$, Mark Arnold ${ }^{4}$, Fabio Garofolo ${ }^{*, 5}$, Mark Bustard ${ }^{6}$, Laura Coppola7, Raj Dhodda ${ }^{8}$, Christopher Evans ${ }^{9}$, Carol Gleason ${ }^{10}$, Sam Haidar ${ }^{11}$, Roger Hayes ${ }^{12}$, Katja Heinig ${ }^{13}$, Noriko Katori ${ }^{14}$, Olivier Le Blaye $^{15}$, Wenkui Li ${ }^{16}$, Guowen Liư ${ }^{4}$ Gustavo Mendes Lima Santos ${ }^{17}$, Min Meng ${ }^{18}$, Bob Nicholson ${ }^{19}$, Natasha Savoie ${ }^{20}$, Michael Skelly ${ }^{11}$, Luis Sojo ${ }^{21}$, Nilufer Tampal ${ }^{6}$, Nico van de Merbel $^{22}$, Tom Verhaeghe ${ }^{23}$, Stephen Vinter ${ }^{24}$, Enaksha Wickremsinhe ${ }^{25}$, Emma Whale ${ }^{24}$, Amanda Wilson ${ }^{26}$, Bärbel Witte ${ }^{27}$ \& Eric Woolf ${ }^{28}$

\section{*Author for correspondence: Tel.: +390691045277 f.garofolo@angelini.it}

Author affiliations can be found at the end of this article.

The views expressed in this article are those of the authors and do not reflect official policy of the US FDA, Europe EMA, Health Canada, France ANSM, The Netherlands MEB, Germany BfArM, Brazil ANVISA, Japan MHWL and UK MHRA. No official endorsement by the FDA, EMA, Health Canada, ANSM, MEB, BfArM, ANVISA, MHWL or MHRA is intended or should be inferred. 


\section{Acronyms}

\begin{tabular}{|c|c|}
\hline Abbreviation & Definition \\
\hline ADME & Absorption, distribution, metabolism, and excretion \\
\hline ANVISA & Brazilian Health Surveillance Agency \\
\hline $\mathrm{APCl}$ & Atmospheric Pressure Chemical Ionization \\
\hline AUC & Area under the curve \\
\hline BMV & Bioanalytical method validation \\
\hline CRO & Contract Research Organization \\
\hline DBS & Dried blood spots \\
\hline EBF & European Bioanalysis Forum \\
\hline EMA & European Medicines Agency \\
\hline ESI & Electrospray ionization \\
\hline IQ & $\begin{array}{l}\text { International Consortium for Innovation and Quality } \\
\text { in Pharmaceutical Development }\end{array}$ \\
\hline $\mathrm{F} / \mathrm{T}$ & Freeze-thaw \\
\hline GCC & Global CRO Council for Bioanalysis \\
\hline HRMS & High resolution mass spectrometry \\
\hline IS & Internal standard \\
\hline ISR & Incurred sample reanalysis \\
\hline ISS & Incurred sample stability \\
\hline LCMS & Liquid chromatography mass spectrometry \\
\hline LLOQ & Lower limit of quantitation \\
\hline MIST & Metabolites in Safety Testing \\
\hline MRM & Multiple reaction monitoring \\
\hline PD & Pharmacodynamic \\
\hline PK & Pharmacokinetic \\
\hline QC & Quality control samples \\
\hline QTOF & Quadrupole time of flight \\
\hline$\%$ RE & Relative error expressed as a percentage \\
\hline SIL-IS & Stable isotope-labeled internal standard \\
\hline SOP & Standard operating procedure \\
\hline SRM & Selected reaction monitoring \\
\hline WRIB & Workshop on Recent Issues in Bioanalysis \\
\hline
\end{tabular}

bioanalytical topics suggested by members of the community, and included presentations from industry leaders and regulatory representatives, culminating in an open panel discussion between the presenters, regulators and attendees in order to determine the consensus items presented in this White Paper.

As with prior WRIB editions [1-9], a significant number of topics were addressed during the workshop and condensed into a series of relevant recommendations. In the present White Paper, the exchanges, consensus and resulting recommendations on 34 recent issues ('hot' topics) in bioanalysis, biomarkers and immuno- genicity are presented. These 34 topics are distributed within the following areas:

Small molecules by LCMS:

- Innovations in small molecule bioanalysis (six topics);

- Regulatory challenges in small molecule bioanalysis (six topics);

\section{Hybrid LBA/LCMS:}

- Innovative method developmentfor biotherapeutics, biomarkers and ADA (five topics);

- Regulatory challenges (three topics);

Large molecules by LBA, biomarkers and immunogenicity:

- LBA bioanalytical challenges (four topics);

- Biomarkers (three topics);

- Immunogenicity (seven topics);

Inputs from regulatory agencies:

- Following the recommendations on the above topics, an additional section of this White Paper focuses specifically on several key inputs from regulatory agencies.

Due to its length, the 2015 edition of this comprehensive White Paper has been divided into three parts. This publication (Part 1) covers the recommendations for small molecule bioanalysis using LCMS. Part 2 (hybrid LBA/LCMS and regulatory agencies' inputs) and Part 3 (large molecule bioanalysis using LBA, biomarkers and immunogenicity) will also be published in volume 7 of Bioanalysis, issues 23 and 24, respectively.

\section{Discussion topics}

Innovations in small molecules by LCMS

Latest advances in high resolution mass spectrometry for small molecule applications: 'it is time to learn method development on HRMS for regulatory submissions'

What is the status of reaching a consensus on the use of high resolution mass spectrometry (HRMS) for regulatory submissions? Are we building on increased industry experience and discussion with regulators on HRMS? Any examples? Was any HRMS submission rejected? If yes, what were the regulators' comments? What are the challenges or differences in method development, validation and sample analysis using 
HRMS in a regulated environment and how are they addressed? Does the extraction window need to be evaluated? Does the mass calibration within batches to correct for drifting need to be evaluated? Has it demonstrated increased selectivity while maintaining good sensitivity? Has this technology demonstrated signal-to-noise enhancement?

\section{How much can we trust ESI in regulated bioanalysis?}

How often do bioanalytical laboratories check for insource reactions during method development when using electrospray? Based on a cursory review of the literature, positive ion electrospray is usually the default ionization mode. How often do laboratories check APCI instead or negative ion electrospray, when feasible?

\section{Beyond 'normal' matrix effects}

Matrix effects can be present in many different forms. Moreover, it can be drug-dependent. Is there a universal solution to avoid matrix effects in bioanalysis? Not all matrix effects, particularly in special population samples, can be foreseen or tested during assay development/validation. Should we be monitoring assay performance only during sample analysis in these cases or is it too late? Can it be done earlier? Should we push for doing stability experiments in fresh plasma or can we rely on ISR (ISS) to pick up stability issues only observed in study (fresh) plasma samples?

Free \& total drug quantification: a challenge for small molecule bioanalysis

For what types of compounds should protein binding be determined during clinical studies? What is the preferred method for protein binding assessment in clinical samples (ultrafiltration or dialysis, etc.)? How are drug concentrations determined in a system in equilibrium without disturbing the equilibrium? What are the quantification challenges of very low free drug concentrations? Free and total methods: is simultaneous quantification possible? What are the method development parameters influencing the unbound/bound drug equilibrium (e.g., $\mathrm{pH}$, temperature, drug concentration, albumin/1-AGP concentration, impact of co-administered drug concentrations)?

\section{Unique/customized method development challenges \& strategies arising from metabolite measurements to fulfill US-FDA \& EU-EMA regulatory requirements}

How often are fit-for-purpose methods versus fully validated methods applied to metabolite quantification to address regulatory requirements? What is the best strategy to deal with unpredictable metabolite instability due to chiral/epimeric/tautomeric/isomeric (Z/E) conversion? Is there an increased use of incurred samples to address metabolite issues (feedback from the recommendations from 2013 and 2014 White Papers in Bioanalysis) [6,7]? Is it currently a regular practice to perform the stability evaluation for metabolites by using incurred samples (ISS)? What is the expert panel opinion on the use of dual sampling in early studies if there is a concern of possible instability but no prior information is available?

\section{Severe method development issues with lipemic} plasma

The impact of lipemic plasma on a bioanalytical assay is increasingly gaining attention by industry and regulatory agencies. Why is the hyperlipidemia evaluation considered significant? Are we compromising data integrity if the evaluation of the impact of hyperlipidemia plasma on quantification is not conducted? Is the understanding of the metabolic pathway critical to determining the need to evaluate and assess the impact of the test? What types of studies are sensitive to potential changes in exposure due to hyperlipidemia? Do we need to be vigilant for studies with oncology indications since inflammation during tumor development alters the cholesterol transport mechanism? What are the regulatory implications of performing and not performing the analysis? Will the companies be at risk of receiving a FDA 483 observation for not evaluating the test if the test is not scientifically warranted? Should hyperlipidemic samples be considered as special population samples and therefore ISR be conducted with such samples?

\section{Regulatory challenges in small molecules by LCMS}

Investigations \& anomalous values with 'no assigned root cause'

What is an anomalous value? When has a root cause been identified? How extensive does an investigation have to be? Where does the bioanalytical laboratory's responsibility stop? Do we need to know the exact root cause for an anomalous value, if we can properly evaluate its impact?

Status of the incurred sample stability evaluation within industry \& regulatory agencies

Incurred sample stability (ISS) has already been exhaustively discussed in the GCC White Paper [10] and 2013 White Paper in Bioanalysis [6]. What is new in 2015? Should incurred samples always be used to support stability (ISS) when spiked QC samples are considered inadequate? If not, why not? According to 


\section{Key term}

Hematocrit: Volume percentage of red blood cells in blood.

the GCC White Paper, ISS may be needed to assess unknown metabolite conjugates, enzymatic degradation of analytes, major matrix instability or oncology studies with co-administered analytes. Do you think ISS should be mentioned in a guidance/guideline even if performed on a case-by-case basis? Are White Papers enough to reinforce the importance of assessing stabilities in incurred samples in the above cases? GCC recommends performing ISS for freeze-thaw stability $(\mathrm{F} / \mathrm{T})$ and short-term/bench-top stability when there are suspected stability issues (case-by-case basis). Should ISS be performed for long-term stability as well? What about for the 2-hr whole blood stability to cover sample collection?

Internal standard variability: new case studies $\&$ regulatory responses from audits

Is there general agreement on the difference between individual variations and systematic differences in response, which may require investigations rather than blindly applying a strict rule? Should we agree on a maximum acceptable difference (and what would it be; $\mathrm{X} \%$ ) for individual variations that should trigger a repeat analysis, or should we leave this open for each laboratory to decide, with enough flexibility to adjust to various situations? What should be the extent required for lab investigations?

\section{Criteria to select weighting factors for linear} \& quadratic calibration curves to answer regulatory agency concerns

Since quadratic regressions can also be used to fit straight-line models, do people feel that the concern over their use is unwarranted? Are people using statistical methods to support the use of quadratic or other regression models or just visual inspection of plots and summaries of $\%$ RE? How are people selecting their weighting function: observation, residual plot assessment, other methods? For linear and quadratic models, do people use weighting factors other than $1 / \mathrm{x}$ or $1 / \mathrm{x}^{2}$ ?

Slope variation in LCMS calibration curves: 'is this an indication of potential method issues?'

Should calibration curve slopes always be consistent for a validated LCMS method, particularly one using a stable isotope labeled compound as internal standard? How likely will calibration curve slope variation lead to a data quality problem? Are calibration curve slopes a good quality control tool? Should significant variation on calibration curve slopes be investigated?
A new life for DBS: 'recent developments

\& advancements'

If the 'hematocrit bias' is managed and the bioanalytical data are accurate does that make DBS 'acceptable?' Would the availability of a device/substrate that will ensure the collection of precise volumes of blood at each sampling time be preferred compared with managing/validating the hematocrit based on a fixed punch size? Would this approach give the regulators more confidence in the data? How much concordance data are needed - between matrices - before one can proceed with DBS as the sole measurement of PK concentrations? It defeats the purpose if dual sampling and dual analysis are needed for each study. What have Pharma/CROs done to minimize dual sampling, while providing enough concordance data to FDA? Is there something we can do to streamline gathering this concordance data (create minimum expectations)? If the hematocrit effect is outside the typical 'acceptance criteria' $(15 / 20 \%)$, would data still be acceptable from special populations such as from neonate studies where the hematocrits can vary outside of this range?

\section{DIscussions, consensus \& conclusions}

\section{Innovations in small molecules by LCMS}

Latest advances in HRMS for small molecule

applications: 'it is time to learn method

development on HRMS for regulatory

submissions'

Triple quadrupole mass spectrometry detectors have long established themselves as the gold-standard instrumentation for small molecule quantitative analysis. When operated in selected or multiple reaction monitoring (SRM/MRM) modes, they offer excellent selectivity and sensitivity with robust, reproducible data. In contrast, high-resolution, accurate-mass mass spectrometry is becoming commonplace for biopharmaceutical analysis, particularly for the analysis of intact molecules. As the demand for more sensitive and selective bioanalytical methods increases, bioanalytical scientists can employ a variety of potentially complex methodologies to deliver the appropriate method. For example, chemical derivatization or modifications to extraction and/or chromatographic procedures can be used. However, sometimes these approaches do not mitigate the selectivity issues that are becoming more prevalent as we move toward lower and lower limits of quantification. The discussion focused on the increased use of HRMS to overcome selectivity and sensitivity challenges.

It was agreed that HRMS boasts some notable improvements as the technology has evolved. These 
include increased sensitivity, faster scan rate, tighter precision and accuracy of methods, improved linear dynamic range and smaller file sizes; the latter having originally been a significant issue. To reduce file size, it is recommended that the instrument be set to collect in MRM mode for QTOF instruments, which contains no extraneous data. In making this recommendation, it was recognized that the loss of qualitative data, which might prove useful at a later time when samples were no longer available to be retested, was unfortunate. However, this consideration is not unique to HRMS, but also for other types of new generation mass spectrometers that are capable of simultaneously collecting both full scan and MRM data (e.g., quantitative/qualitative approaches on latest generation triple quadrupoles and linear ion traps). Hence, for HRMS as for triple quadrupole and ion traps, when used in quantitative regulated bioanalysis, it is recommended not to acquire extra data that may not be used to answer the immediate objectives of the study.

It was a unanimous consensus that in HRMS, sensitivity is improved because of reduced background noise, which improves the signal-to-noise ratio so that a low LLOQ can be achieved even for very complex matrices [11]. Sensitivity is also gained for molecules found to have poor MRM fragmentation on standard triple quadrupole mass spectrometers. It is recommended to carefully evaluate the HRMS QTOF mass drift in method development, adding specific tests during method validation; drift can then be subsequently monitored during sample analysis using, if possible, a SIL-IS.

Although not yet widely used, but with swiftly gaining popularity, regulated studies supported using HRMS have already been submitted to regulatory agencies and no concerns were raised. Hence, the overall recommendation from industry opinion leaders who are using this platform is that HRMS can be used for studies submitted to regulatory agencies provided that the equipment is compliant for use in a regulated environment, as the validation and sample analysis expectations using HRMS are largely similar to those of traditional triple quadrupole instruments.

\section{How much can we trust ESI in regulated bioanalysis?}

Electrospray ionization mass spectrometry is the detection method of choice in present-day bioanalysis of drugs and their metabolites. A large number of molecules have basic moieties that are readily protonated; therefore, positive ion ionization is the most popular mode. However, because of the electrochemical nature of electrospray ionization, positive electrospray could help to predict potential oxidation species of a compound [12,13].

It has been agreed that although these reactions can be exploited to understand and provide a forward look into the potential for an analyte to oxidize in solution, this occurrence can affect both qualitative and quantitative analysis. Examples are available where not only oxidation can affect analysis [14], but ESI can also facilitate the formation of covalent dimers through oxidative coupling in the ion source [15] or the formation of adduct ions [16].

It was acknowledged that, from a method development point of view, it is beneficial to understand these processes and where possible, control them or eliminate them completely, particularly when other means of ionization are not feasible. It is recommended to avoid simply relying on an autotune approach to identify the parent ion since this can lead to the absence of important information. The standard practice recommended is to infuse the compound into the mass spectrometer and evaluate the presence of parent and product ions. It is also valuable to expand the mass window to detect possible adducts and dimers and perform a manual tuning of the system. The main goal is to understand what ions are being produced and to adjust the method accordingly.

\section{Beyond 'normal' matrix effects}

Bioanalytical plasma assays are developed based on the assumption that the blank matrix used for developing and validating the assay is representative of the matrix that will be obtained from the subjects participating in the study. However, study samples come from a variety of subjects including patients and healthy volunteers, with a multitude of possible differences. Furthermore, matrix effects tend to be drug dependent. It was unanimously agreed that it is not feasible to test every possible matrix type during assay development or validation; therefore, it is necessary to build mitigation plans into the method to detect and minimize the impact of potential unexpected matrix effects. Robust method development, good chromatography and the use of a SIL-IS are key to producing reliable bioanalytical methods.

Despite these safety nets, it has been agreed that it is not possible to reliably predict the potential impact of matrices from special populations. As such, it is recommended to continuously monitor IS response during sample analysis for any anomalies or variations that could indicate an unpredictable matrix effect. If detected, an investigation should be initiated in order to determine its impact, which may include dilution of the sample with control matrix or the use of a pre-dose sample for QC preparation to assess the compensation of matrix effects by the IS. 
It was also agreed that another point of concern regarding the matrix of special populations is the possibility of differences in stability of the drug when using fresh matrix versus frozen or stored matrix. It was discussed whether there should be a push for doing stability experiments in fresh plasma, and how fresh plasma should be defined, for example by measuring esterase activity. It was concluded that closely monitoring ISR is an important requirement for studies with special populations [17]. While ISR is not intended to monitor stability, any stability issues detected during the ISR evaluation could lead to an ISS investigation as well.

Free \& total drug quantification: a challenge for small molecule bioanalysis

Plasma protein binding refers to non-covalent interactions of a drug with plasma proteins like albumin or $\alpha 1$-acid glycoprotein. Protein binding determinations can be affected by factors such as $\mathrm{pH}$ and temperature, as well as conditions such as renal insufficiency, which can impact protein binding in vivo.

Following administration, drugs in circulation establish equilibrium between free and plasma protein bound species. The degree of protein binding is generally dependent upon the chemical properties of the species. The desire to quantitate the concentration of 'free drug' is grounded in the belief that the pharmacological properties of a given drug are based primarily on the amount of unbound drug in circulation. The foundation of this concept is the assertion that only unbound drug is 'bioavailable' to partition into the site of drug action and interact with the molecular target.

Various techniques including equilibrium dialysis, ultrafiltration and ultracentrifugation have been developed to assess plasma protein binding, each with their pros and cons. It was agreed that ultrafiltration is useful because it can easily be implemented at clinical sites, as there are no unique equipment requirements. However, nonspecific binding can be an issue with this method; the analyte may bind to the device/membrane and the ultrafiltrate can behave more like urine than plasma, which itself has more associated bioanalytical issues. Ultracentrifugation has none of the membrane problems seen in ultrafiltration; however, it does require specialized equipment and is very slow. Furthermore, lipids in the supernatant liquid may bias results. It was agreed that the current gold standard for protein binding assessments is equilibrium dialysis. However, this method is not without challenges including the requirement for long incubation times and errors caused by protein breakthrough of the ultrafiltration membrane.

It was concluded that the usefulness of both free and total drug quantification for typical clinical studies is debatable. These results are typically used during drug discovery as part of the process of ranking viable compounds. However, for compounds with binding greater than $99 \%$, the sensitivity, accuracy and precision of the analytical method used to assess protein binding becomes critical. It was recommended that assessment of binding is needed in specific situations, for example renally cleared drugs that are excreted unchanged, narrow therapeutic index drugs and other special situations (e.g., understanding the effectiveness of antidotes in binding free drug) [18]. FDA confirmed that submissions typically included data for total drug concentrations (combined free and protein bound). A subtraction to arrive at a bound concentration from free and total concentrations is not generally acceptable, making equilibrium dialysis quite important.

\section{Unique/customized method development challenges \& strategies arising from metabolite measurements to fulfill US-FDA \& EU-EMA regulatory requirements}

The measurement of metabolites is often a necessary and integral part of a clinical study, as indicated in the MIST guidance [19], which outlines the need to demonstrate safety coverage of major and unique human metabolites. It is necessary to determine if metabolites significantly contribute to the overall pharmacological activity of the drug or if they are the mediators of drug-drug interactions. It is also essential to elucidate clearance pathways of drugs when measured in excreta [20].

It was agreed that in general, three approaches with different extents of validation could be employed in the bioanalysis of metabolite(s) of drug candidate(s) in biological samples to meet the expectations of health authorities. These approaches include: (1) implementation of validated methods that follow the current regulatory guidance and industry best practice for generating reproducible bioanalytical concentration data, (2) quantitative analysis of study samples using qualified methods for generating concentrations of metabolite(s) of interest, and (3) semi-quantitative analysis of given species samples by assessing relative instrument response to estimate concentrations of metabolite(s) of interest.

Depending on the stage of drug development and the purpose of the study, the approaches outlined above can be employed for the analysis of pooled study samples from selected time points according to AUC pooling or individual toxicokinetic or pharmacokinetic samples collected in preclinical or clinical studies. It is common practice - based on the MIST guideline [19] - that only significant and unique human metabolites are followed throughout drug development. 
It was agreed that, typically, qualified methods or semi-quantitative methods (otherwise known as fitfor-purpose methods) are sufficient for early MIST assessments. Active metabolites that contribute to the effectiveness of the drug are expected to be measured throughout the program using fully validated methods. For major inactive metabolites, the only ones that need to be followed are those that do not have adequate exposure coverage within the animal toxicology species. For chiral, epimeric, tautomeric or isomeric compounds, conversion and time dependence should be assessed; it is important to assess the metabolic pathway to categorize the transient and nontransient species.

In light of the recommendations from the 2013 and 2014 White Papers in Bioanalysis [6,7], discussions explored whether there was an increased use of incurred samples to investigate metabolites. It appeared that incurred samples are generally used during investigations, on a case-by-case basis, and that understanding the metabolism of the drug might help reduce the use of these samples.

Regardless of which type of bioanalytical method is used and regardless of the purpose of the study, stability of both the parent drug and its metabolite(s) in the intended study sample matrix needs to be assessed and controlled. The use of incurred animal samples to test drug and metabolite stability is a common practice. Moreover, failure to preserve an unstable drug molecule and/or its metabolite(s) in study sample collection, processing, storage, extraction and detection can lead to under- or overestimation of drug/metabolite concentrations. If there is a concern of possible instability (e.g., freeze-thaw stability issue, instability under room temperature) but no prior information is available, dual sampling (with and without stabilizer addition) in early clinical studies is suggested as an option; however, single sample analysis remains the default. Dual samples can be collected when appropriate.

\section{Severe method development issues with lipemic plasma}

The impact of lipemic plasma on bioanalytical assays has increasingly gained the attention of industry and regulatory agencies over the last 10 years. The first agency to address the issue was ANVISA in 2003, and the topic has since been incorporated into the EMA and ANVISA validation guidance documents [17,21]. Since 2012, FDA has also begun to focus on this evaluation during regulatory inspections.

It was agreed that the science behind the drive for assessing the impact of lipemic plasma in validation is reasonably clear. Lipids such as phosphatidyl choline and mostly all forms of triglycerides have the poten- tial to cause significant matrix effects. Considering that the American Heart Association reported elevated cholesterol concentrations in a significant portion of the American population [22], there is an increased likelihood that some bioanalytical study samples will be hyperlipidemic. Therefore, the effect of lipids on the performance of the method should be evaluated during method development.

Lipemic interference can be caused by physical or chemical effects or by volume displacement effects. Interestingly, it is also reported that the proportion of lipids to water in plasma is significantly different (typically $8 \%$ in normal plasma versus up to $25 \%$ in lipemic plasma [23]), which can affect the distribution of the drug depending on its hydrophobicity. It was agreed that homogeneity of the sample is an important factor to consider during assessment of lipemic interference. Hence, it was recommended that the greatest consideration of potential lipemia and its impact on the mechanics of the method should be evaluated during method development, for example effects on cartridge or column flow obstruction. Finally, preliminary evidence shows that physicochemical properties of a compound contribute significantly to the precision and accuracy of the quantification of analytes in hyperlipidemic plasma.

According to the audience and panelists, there are very few examples of lipemia effects. However, a few do exist [24-26]. It was agreed that the evaluation should not be required for preclinical studies where controlled diets are the norm, unless the drug impacts lipid metabolism or the particular strain of animals has been bred to have abnormal plasma lipid levels.

\section{Regulatory challenges in small molecules by LCMS}

Investigations \& anomalous values with 'no assigned root cause'

The goal of bioanalytical method validation is to demonstrate that a bioanalytical method reliably determines analyte concentrations in a given biological matrix. Despite the application of good science in method development, it is possible that abnormal results can occur during method validation and later during sample analysis. Industry and regulators concurred that when unexpected or spurious results arise, thorough and documented investigations are expected. Evidence of this opinion can be seen in the guidance documents and White Papers where investigations and abnormal results are mentioned [2,17,27-29].

It was agreed that the difficulty lies in the often subjective evaluation in defining an anomalous or abnormal result and what constitutes a thorough 
investigation. A simplistic, yet logical, definition of an anomalous result is an unexpected event which results in values that 'do not make sense,' that is, biologically implausible results. Moreover, regulatory agencies also look for investigations into high failure rates. A distinction between unexpected results of calibration standards or QC samples and study samples is needed, since unusual results for samples of known concentration are in general easier to detect and investigate. Some examples of unexpected results include ISR failures, analyte peaks in predose samples (period 1, or in later periods where the presence of peaks cannot be explained by long terminal elimination of the drug of interest), concentration-time profiles with multiple Cmax values, apparent outliers (samples where the determined concentrations just 'do not seem to fit the profile' compared with prior and subsequent samples) and chromatographic interferences present only in study samples. It was agreed that in these situations, an investigation is needed which could include reanalysis of selected samples. The process for selection of these samples should be defined a priori by SOPs in compliance with current regulatory requirements. Original and repeat values should be tabulated and presented in the bioanalytical study report.

It was recommended that the extent of investigation is determined on a case-by-case basis, taking into consideration the impact of the anomalous event and the frequency of failures. This criterion should be SOP driven. The goal of the laboratory investigation is to find a plausible or probable explanation, placing importance on the preliminary investigation data addressing sample handling and other analytical events. Ultimately, bioanalytical laboratories should be obliged to follow up if the investigation is subsequently deemed inadequate by regulators. Scientific justification and plausibility should be communicated to all parties involved in the clinical development program.

Often the root cause for an event cannot be determined; therefore, the need to continue the investigation can come into question. The cause may be study independent and the frequency needs to be evaluated to assess the impact of the event. Regulators often look for systematic causes and not isolated ones. Therefore, it was recommended to perform, document and report a thorough impact assessment and to ultimately demonstrate with reasonable certainty that systematic issues did not occur.

Status of the incurred sample stability evaluation within industry \& regulatory agencies

The topic of ISS continues to stimulate discussion within the bioanalytical community. The subject was discussed at length in the 2013 and 2014 White Papers in Bioanalysis [6,7], and it was the topic of a specific GCC White Paper [10]. In this document shared with worldwide regulatory agencies, GCC recommended that ISS experiments should not be conducted routinely, but on a case-by-case basis when there are questions of analyte stability in incurred samples. For example, GCC suggests that ISS experiments may be useful when backconversion of unknown metabolite conjugates may be present, there may be enzymatic degradation of analytes, there are indications of major matrix instability, spiked QC samples are unable to demonstrate stability or to demonstrate stability with co-administered drugs for oncology studies. At this time, it was agreed that formal regulatory guidance is not required since these questions arise from specific, individual cases. However, consensus was reached that potential risks on stability should be evaluated and documented during method development and/or method validation.

The recommendation that fortified QC samples of known concentration be used, outlined in previous White Papers $[6,7,10]$, should continue unless sound scientific judgment suggests otherwise. QC samples are still the industry standard to prove long-term matrix stability. However, inconsistent results in the analysis of repeats, for example ISR testing performed at the beginning, middle and end, would likely trigger a more thorough ISS investigation.

If ISS is used to further investigate stability questions, the GCC White Paper recommends that freeze-thaw and bench-top ISS evaluations should be performed. It was agreed to extend the recommendation and consider long-term and whole blood stability assessments if appropriate. Regulators encouraged industry to continue to document and to report cases where ISS issues are discovered.

Internal standard variability: new case studies \& regulatory responses from audits

Discussions and recommendations surrounding IS variation at the WRIB goes back as far as the 2011 White Paper in Bioanalysis [4] and a GCC White Paper [29]. Consensus among the industry and regulators has not been reached.

It was stated during the discussion by regulatory agency representatives that, in studies considered as pivotal to market authorization, criteria in SOPs should define the acceptance limits of IS responses and should define procedures to be followed in cases of IS response failure. It was agreed that variations in IS response are expected to some extent during the analysis of bioanalytical samples. Many laboratories have SOPs with acceptance limits for internal standard response variation within a run, such as a percentage of the mean response. Such criteria can indeed help to identify inter- 
nal standard spiking mistakes, such as double spikes or incomplete IS volume transfers during sample processing, which can affect accuracy of the measurement. Although regulators do not recommend a particular acceptance range, many in industry find the $50-150 \%$ range of the mean response to be useful. Another approach is to base acceptance criteria on the variability in IS response observed during method validation.

It was agreed that it can be difficult to describe and define in SOPs when trends or systemic differences occur in a bioanalytical run, and whether they affect concentration results. In some cases, IS variability is a characteristic of the method, and the impact of the variations is assessed during method validation with precision and accuracy data. Other systemic problems may not be seen until routine sample analysis, and these may be unpredictable. Practical examples include differences between study samples and standard and/or QC samples, or gradual decreases or increases in IS response during runs with the last QCs failing to meet acceptance criteria. Acceptance criteria such as percentage of the mean IS response are not always able to handle such situations. ISR data have helped to determine the influence of internal standard variations on the reliability of results [30]. The goal is not to detect individual outliers, but rather detect trends in unknown samples. It was acknowledged that there is a difference between individual variations and systemic differences in responses. It was recommended to evaluate trends and systemic differences. It was suggested that IS responses for each run should be plotted and included with the raw data for each batch. These data need not be presented in the bioanalytical report but upon review may assist the bioanalyst in triggering an investigation based on unexpected IS responses in order to identify their root cause and assess whether the accuracy of calculated concentrations is affected. These investigations may conclude that certain analytical runs need to be rejected, resulting in reanalysis of study samples. It was agreed that such decisions should be science-driven and their rationale should be clear and well documented. The method and extent of investigations should not be prescribed by regulatory guidance but procedures need to ensure that the IS tracks the analyte response in incurred samples throughout analysis. The ultimate objectives should be evaluating the accuracy of the method and the need for further investigations.

\section{Criteria to select weighting factors for linear \& quadratic calibration curves to answer regulatory agency concerns}

Global regulatory authorities continue to evaluate documentation for the choice of regression model and weighting factor for LCMS bioanalysis calibra- tion functions. Most bioanalytical method validation guidelines $[17,27,28]$ recommend and many regulations [21] request the simplest model. The definition of 'simplest' has already been discussed in the 2008 White Paper in Bioanalysis [1]. However, further discussion was deemed necessary since it seems to be a term that is applied differently across the industry.

It was discussed that since quadratic regressions can also be used to fit straight-line models, concern over their use may be unwarranted. However, it was agreed that selection of the regression model should be step by step, beginning with a simple model, like linear, and moving to more complex models, like quadratic or weighted models. Providing that its successful use is demonstrated during method validation, the quadratic regression type can be acceptable in many cases.

There are simple statistical approaches to selecting simple and defensible regression models with appropriate weighting. It was recommended that the best fit should be selected with a predefined statistical approach, documented as part of company SOPs, for example lowest sum of residual error, a general test for goodness of model fit, significance of the quadratic term added to a linear model, residual plots for investigation of weight schemes, etc. An informal poll of the attendees indicated that the most-used weighting factors were $1 / x$ and $1 / x^{2}$.

Slope variation in LCMS calibration curves: 'is this an indication of potential method issues?'

A calibration curve established using known standard samples is the basis for most quantitative bioanalytical analysis. The quality of the calibration curve predicts the accuracy of the quantitative measurements. In regulated bioanalysis using LCMS, the calibration curve slope can be a quality indicator for assay performance. It was agreed that a consistent calibration curve slope across multiple analytical runs is a reassuring sign that the assay is rugged and reliable. Although there is no regulation on performance of calibration slope during method validation and sample analysis in regulated bioanalysis, irreproducibility and imprecision of the assay can often be attributed to slope variations.

There is more than one reason for variation in calibration curve slopes, even when a SIL-IS is used [31]. For example, matrix effects and detector saturation [32], nonspecific adsorption [33] or differential recoveries [34] can cause differences in slopes when using a SIL-IS.

It was agreed that slopes of analytical batches conducted on the same instrument should be consistent. Slopes will be more variable across multiple instruments due to variabilities caused by system maintenance and cleaning and differences in initial instrument setup. However, it is recommended to investigate 


\section{Key term}

Microsampling: Very small volumes of blood (typically

much less than $100 \mu \mathrm{l}$ ) collected from humans or animals.

unexplained, extreme variations of slope, even with batches that meet the acceptance criteria, to determine if data remain precise and accurate.

\section{A new life for DBS: 'recent developments}

\section{\& advancements'}

DBS sampling may be an alternative means for collecting blood during preclinical and clinical studies. Discussions and extensive recommendations on this subject have already been reported in two recent White Papers [5,35]. However, regulatory guidelines for DBS utilization have not yet been developed, although there is demand [36]. Currently, use of DBS as the sole method of sampling is approved on a case-by-case basis by regulators, and generally involves bridging data to prove concordance prior to clinical development [35,37]. There is no current consensus on how much concordance data are needed, or if they are necessary if the DBS assay has been appropriately validated.

The discussion during the workshop confirmed that the ability to collect small sample volumes combined with the simplicity in collecting, shipping and storage made DBS a practical and popular microsampling technique, but its broader implementation has been slow and limited. The main driver behind the implementation of DBS sampling for clinical trials was most likely the significant clinical advantages (ease of implementation globally, across multiple sites, remote sites that may lack extensive instrumentation and infrastructure to generate plasma samples and their storage, and savings in shipping costs).

It was confirmed that the change of matrix from plasma to blood introduces a variable in comparing to the standard plasma method and this requires understanding the partitioning/association of the drug to red blood cells [38]. Additionally, impact of hematocrit variation on the spot sampling method and resulting concentration bias, commonly termed the 'hematocrit effect,' continues to raise concerns across industry and regulators. However, it was agreed that progress is being made in sample collection to alleviate or eliminate the hematocrit effect, especially with the availability of techniques and devices that ensure the collection of precise volumes of blood at each sampling time. DBS analysis is also impacted by analytical challenges such as the LLOQ (due to the small sample volume) and sample throughput (lack of automation) that are being mitigated by recent advancements in instrumentation. Although advancements have been made to minimize or eliminate the hematocrit bias with DBS sampling, concerns have also been raised with regard to the age and extractability of the spot, and the timing and mode of application of the IS.

It was concluded that DBS is not a panacea and is not expected to replace plasma but should be a component in the bioanalyst's tool-box (to be used when needed based on program needs). Moreover, DBS sampling in clinical programs should be considered in agreement with regulatory agencies, especially when it offers specific advantages such as the simplification of sample collection, in-home sampling, use in vulnerable populations (e.g., neonates, elderly, oncology patients).

\section{Recommendations}

Below is a summary of the small molecules by LCMS recommendations made during the 9th WRIB.

\section{Innovations in small molecules by LCMS}

- HRMS has been shown to possess excellent precision and accuracy and can be used to support regulated studies as long as the equipment is suitable for use in a regulated environment. Method validation expectations are similar to those in typical triple quadrupole mass spectrometry methods. Drift in the mass analyzer should be evaluated during method development and validation, and then subsequently monitored during sample analysis using a SIL-IS.

- When using ESI, it is best to understand the ionization reactions and to adjust the method accordingly. The use of the autotune approach alone is not recommended for ESI. Recommended practice is to infuse the compounds into the mass spectrometer and identify precursor and product ions characteristic of the analytes. It is also considered standard to expand mass windows and to perform a manual tuning of the system.

- Since it is not feasible to test every population matrix type during assay development or validation, SIL-IS response should be monitored during sample analysis for anomalies that could indicate unanticipated matrix effects. If IS anomalies are observed, an investigation should be initiated. The impact of matrix effects may be assessed by diluting samples with control matrix or using a predose sample for QC preparation to assess the ability of the IS to compensate. Moreover, potential instability questions identified during ISR experiments should lead to a thorough ISS investigation.

- Regulatory submissions primarily focus on the analysis of total drug concentrations. Assessment 
of binding is most appropriate for renally cleared drugs that are excreted unchanged, narrow therapeutic index drugs, special populations (renally or hepatic impaired) and other special situations.

- Qualified and semi-quantitative methods are sufficient for early MIST assessments. Assessments of active and significant human metabolites should use fully validated methods. No regulatory expectations to assay major, inactive metabolites exist for those with adequate coverage in toxicology species unless a problem is anticipated in special populations. It is best to assess conversion and time dependence for chiral, epimeric, tautomeric or isomeric compounds. It is best to assess and control stability of both the parent drug and its metabolite(s) in the intended study sample matrix. Single samples remain the default for these assessments. Dual samples can be collected when there is a concern of possible instability.

- Homogeneity of lipemic samples is important to consider during the assessment of lipemic interference. Physicochemical property of a compound may impact the precision and accuracy of the quantification of the analytes in hyperlipidemic plasma. Care should be exercised during hyperlipidemia evaluation in hemolyzed samples. The evaluation should not be required for preclinical studies unless the drug impacts lipid metabolism or the particular animal strain is hyperlipidemic.

\section{Regulatory challenges in small molecules by LCMS}

- Thorough and documented investigations of anomalous results, including an assessment of impact on the data, are expected. Samples with anomalous values selected and repeated by the lab should be defined a priori by SOPs and in compliance with current regulatory requirements. The extent of an investigation should be SOP-driven and aimed at identifying both systemic and isolated incidents.

- ISS experiments should not be conducted routinely, but on a case-by-case basis when specific analyte stability questions are suspected in incurred sample results. QC samples of known concentration to represent study samples should continue as the performance measure unless sound scientific judgment suggests otherwise. Analysis of repeats including ISR testing performed at the beginning, middle and end of analysis by using samples stored for different durations can be an indicator of potential instability in incurred samples and can trigger a more elaborate ISS investigation. Freeze-thaw, bench-top, long-term and whole blood ISS evaluations should be performed when ISS investigations are required, although in unique cases where problems with ISS persist additional ISS/stability evaluations may be justified.

- Variations in IS response should be mitigated by use of a SIL-IS. SOPs with acceptance limits for IS response variation should be established to identify technical problems during sample processing. Trends and systemic differences should be investigated to identify their root causes and to determine effects on the accuracy of the drug/analyte concentration results in matrix. Investigations should be science-driven with clear rationale and documentation.

- Selection of the regression model should be step by step, beginning with a simple model (linear) and then moving to more complex models (quadratic) each testing the model improvement through the use of weighting, supported by a predefined statistical approach and documented as part of company SOPs.

- Slopes of batches analyzed on the same instrument should be consistent. Unexplained, extreme variations should be evaluated to assess impact on accuracy.

- Most regulatory agencies recommend bridging data to establish concordance between DBS and traditional plasma assays prior to implementing DBS as the sole method of sampling in the clinical development. The utilization of DBS sampling in clinical programs should be discussed in concert with regulatory agencies when it offers specific and well-defined advantages.

\section{Acknowledgements}

The authors would like to acknowledge the US FDA, Europe EMA, France ANSM, The Netherlands MEB, UK MHRA, Germany BfArM, Brazil ANVISA, Health Canada and Japan MHWL for supporting this workshop. E Fluhler (Pfizer), J Welink (EMA/Dutch MEB), B Ackermann (Eli Lilly), N Hughes (Life Labs), F Garofolo (Angelini Pharma), A Song (Genentech), T Thway (Amgen), L Amaravadi (Biogen Idec) and H Myler (Bristol-Myers Squibb) for chairing the workshop and/or the White Paper discussions. All the workshop attendees and members of the bioanalytical community who have sent comments and suggestions to complete this White Paper. W Garofolo, L Lu, X Wang, M Losauro, N Savoie, A Hernandezand, S Schonert, D Cohen, K Kalaydjian, P de Souza for the assistance in the organization of the event. Future Science Group as a trusted partner. 
Financial \& competing interests disclosure

The authors have no relevant affiliations or financial involvement with any organization or entity with a financial interest in or financial conflict with the subject matter or materials discussed in the manuscript. This includes employ-

\section{Author affiliations}

'Dutch MEB, Utrecht, The Netherlands

2Pfizer, Pearl River, NY, USA

${ }^{3}$ Bioanalytical Laboratory Services a Division of LifeLabs LP, Toronto, ON, Canada

${ }^{4}$ Bristol-Myers Squibb, Princeton, NJ, USA

${ }^{5}$ Angelini Pharma, Piazzale della Stazione, snc, 0040 S. Palomba

Pomezia (RM), Italy

${ }^{6}$ Health Canada, Ottawa, ON, Canada

${ }^{7}$ Apotex, Toronto, ON, Canada

${ }^{8}$ AbbVie Inc., North Chicago, IL, USA

${ }^{9}$ GlaxoSmithKline, King of Prussia, PA, USA

${ }^{10}$ Bristol-Myers Squibb, Tully, NY, USA

"US FDA, Silver Spring, MD, USA

${ }^{12} \mathrm{MPI}$ Research, Mattawan, MI, USA

${ }^{13}$ F. Hoffmann-La Roche Ltd., Basel, Switzerland

\section{References}

1 Savoie N, Booth BP, Bradley T et al. 2008 White Paper: the 2nd Calibration and Validation group workshop on recent issues in good laboratory practice bioanalysis. Bioanalysis 1(1), 19-30 (2009).

2 Savoie N, Garofolo F, van Amsterdam P et al. 2009 White Paper on recent issues in regulated bioanalysis from the $3 \mathrm{rd}$ Calibration and Validation group workshop. Bioanalysis 2(1), 53-68 (2010).

3 Savoie N, Garofolo F, van Amsterdam P et al. 2010 White Paper on recent issues in regulated bioanalysis and global harmonization of bioanalytical guidance. Bioanalysis 2(12), 1945-1960 (2010).

4 Garofolo F, Rocci M, Dumont I et al. 2011 White Paper on recent issues in bioanalysis and regulatory findings from audits and inspections. Bioanalysis 3(18), 2081-2096 (2011).

5 DeSilva B, Garofolo F, Rocci M et al. 2012 White Paper on recent issues in bioanalysis and alignment of multiple guidelines. Bioanalysis 4(18), 2213-2226 (2012).

6 Stevenson L, Rocci M, Garofolo F et al. 2013 White Paper on recent issues in bioanalysis: 'hybrid' - the best of LBA and LCMS. Bioanalysis 5(23), 2903-2918 (2013).

7 Fluhler E, Hayes R, Garofolo F et al. 2014 White Paper on recent issues in bioanalysis: a full immersion in bioanalysis (Part 1 - small molecules by LCMS). Bioanalysis 6(22), 3039-3049 (2014).

8 Dufield D, Neubert H, Garofolo F et al. 2014 White Paper on recent issues in bioanalysis: a full immersion in bioanalysis (Part 2 - hybrid LBA/LCMS, ELN \& regulatory agencies' input). Bioanalysis 6(23), 3237-3249 (2014).

9 Stevenson L, Amaravadi L, Myler H et al. 2014 White Paper on recent issues in bioanalysis: a full immersion in bioanalysis (part3 - LBA and immunogenicity). Bioanalysis 6(24), 3355-3368 (2014). ment, consultancies, honoraria, stock ownership or options, expert testimony, grants or patents received or pending, or royalties.

No writing assistance was utilized in the production of this manuscript

\author{
${ }^{14}$ Japan MHLW-NIHS, Tokyo, Japan \\ ${ }^{15}$ France ANSM, Saint-Denis, France \\ ${ }^{16}$ Novartis, East Hanover, NJ, USA \\ ${ }^{17}$ Brazil Anvisa, Brasilia, Brazil \\ ${ }^{18}$ Covance, Salt Lake City, UT, USA \\ ${ }^{19} \mathrm{PPD}$, Richmond, VA, USA \\ ${ }^{20}$ CFABS, Montreal, QC, Canada \\ ${ }^{21}$ Xenon Pharmaceuticals Inc., Burnaby, BC, Canada \\ 22PRA Health Sciences, Assen, The Netherlands \\ ${ }^{23}$ Janssen Research \& Development, Beerse, Belgium \\ ${ }^{24}$ UK MHRA, London, UK \\ ${ }^{25}$ Eli Lilly \& Company, Indianapolis, IN, USA \\ ${ }^{26}$ AstraZeneca, Cambridge, UK \\ ${ }^{27}$ Germany BfArM, Bonn, Germany \\ ${ }^{28}$ Merck Research Labs, West Point, PA, USA
}

10 Lowes S, LeLacheur R, Shoup R et al. Recommendations on incurred sample stability (ISS) by GCC. Bioanalysis 6(18), 2385-2390 (2014).

11 Voelker T, Wang H, Irish M et al. Method development and validation of six bile acids for regulated bioanalysis: improving selectivity and sensitivity. Bioanalysis 5(10), 1229-1248 (2013).

12 Van Berkel GJ, Kertesz V. Utilizing the inherent electrolysis in a chip-based nanoelectrospray emitter system to facilitate selective ionization and mass spectrometric analysis of alkylporphyrins. Anal. Bioanal. Chem. 403, 335-343 (2012).

13 Plattner S, Erb R, Chervet JP et al. Ascorbic acid for homogenous redox buffering in electrospray ionization-mass spectrometry. Anal. Bioanal. Chem. 404(5), 1571-1579 (2012).

14 Karancsi T, Slégel P, Novak K et al. Unusual behaviour of some isochromene and benzofuran derivatives during electrospray ionization. Rapid Comm. Mass Spectrom. 11, 81-84 (1997).

15 Sojo LE, Chahal N, Keller BO. Oxidation of catechols during positive ion electrospray mass spectrometric analysis: evidence for in-source oxidative dimerization. Rapid Comm. Mass Spectrom. 28, 2181-2190 (2014).

16 Guaratini T, Vessecchi R, Pinto E et al. Balance of xanthophylis molecular and protonated molecular ions in electrospray ionization. J. Mass Sprectrum. 40, 963-968 (2005).

17 European Medicines Agency. Committee for Medicinal Products for Human Use (CHMP). Guideline on bioanalytical method validation. London, UK (2011). EMEA/ CHMP/EWP/192217/2009. www.ema.europa.eu/docs/en_GB/document_library/ Scientific_guideline/2011/08/WC500109686.pdf

18 Benet LZ, Hoener BA. Changes in plasma protein binding have little clinical relevance. Clin. Pharm. Ther. 71(3), 115-121 (2002). 
19 US FDA. Guidance for industry: safety testing of drug metabolites (2008).

www.fda.gov/downloads/Drugs/

GuidanceComplianceRegulatoryInformation/Guidances/ ucm079266.pdf

Ma S, Chowdhury SK. A tiered approach to address regulatory drug metabolite-related issues in drug development. Bioanalysis 6(5), 587-590 (2014).

21 ANVISA. Resolution RDC no 27, May 27, 2012 Brazilian Health Surveillance Agency, Brazil (2012).

22 Mozaffarian D, Benjamin EJ, Go AS et al. Heart disease and stroke statistics-2015 update: a report from the American Heart Association. Circulation 131(4), e29-e322. (2014).

23 Nikolac N. Lipemia: causes, interference mechanisms, detection and management. Biochem. Med. (Zagreb.) 24(1), 57-67 (2014).

24 Martinez-Subiela S, Ceron JJ. Effect of hemolysis, lipemia, hyperbilirubinemia and anticoagulants in canine $\mathrm{C}$-reactive protein, serum, amyloid A and ceruloplasmin assays. Can. Vet. J. 7, 625-629 (2005).

25 Henderson RJ, Wasan KM, Leon CG. Haptoglobin inhibits phospholipid transfer protein activity in hyperlipidemic human plasma. Lipids Health Dis. 8(27), 1-8 (2009).

26 Chin C, Zhang ZP, Kames HT. A study of matrix effects on an LC/MS/MS assay for olanzapine and desmethyl olanzapine. J. Pharm. Biomed. Anal. 35, 1149-1167 (2004).

27 US Department of Health and Human Services, US FDA, Center for Drug Evaluation and Research, Center for Veterinary Medicine. Draft guidance for industry, bioanalytical method validation, Rockville, MD, USA (2013). www.fda.gov/downloads/drugs/ guidancecomplianceregulatoryinformation/guidances/ ucm368107.pdf

28 US Department of Health and Human Services, US FDA, Center for Drug Evaluation and Research, Center for Veterinary Medicine. Guidance for industry, bioanalytical method validation, Rockville, MD, USA (2001). www.fda.gov/downloads/Drugs/.../Guidances/ucm070107. pdf
29 Lowes S, Jersey J, Shoup R et al. Recommendations on: internal standard criteria, stability, incurred sample reanalysis and recent 483 s by the Global CRO Council for Bioanalysis. Bioanalysis 3(12), 1323-1332 (2011).

30 Tan A, Hussain S, Musuku A, Massé R. Internal standard response variations during incurred sample analysis by LC-MS/MS: case by case trouble-shooting. J. Chromatogr. B. 877(27), 3201-3209 (2009).

31 Zhao Y, Liu G, Shen JX, Aubry AF. Reasons for calibration standard curve slope variation in LC-MS assays and how to address it. Bioanalysis 6(11), 1439-1443 (2014).

32 Liu G, Ji QC, Arnold ME. Identifying, evaluating, and controlling bioanalytical risks resulting from nonuniform matrix ion suppression/enhancement and nonlinear liquid chromatography-mass spectrometry assay response. Anal. Chem. 82(23) 9671-9677 (2010).

33 Gu H, Deng Y, Wang J et al. Development and validation of sensitive and selective LC-MS/MS methods for the determination of BMS-708163, a $\gamma$-secretase inhibitor, in plasma and cerebrospinal fluid using deprotonated or formate adduct ions as precursor ions. J. Chromatogr. $B$. 878(25), 2319-2326 (2010).

34 Wieling J. LC-MS-MS experiences with internal standards. Chromatographia Suppl. 55(1), S107-S113 (2002).

35 Evans C, Arnold M, Bryan P et al. Implementing dried blood spot sampling for clinical pharmacokinetic determinations: considerations from the IQ consortium Microsampling Working Group. AAPS J. 17(2), 292-300 (2015).

36 Viswanathan CT. Perspectives on microsampling: DBS. Bioanalysis 4(12), 1417-1419 (2012).

37 Dockendorf M, Li CC, Kowalski K et al. Population modeling is a critical element of bridging pharmacokinetic (PK) data from dried blood spot and plasma across clinical programs. Presented at: 2014 AAPS Annual Meeting. San Diego, CA, USA, 2-6 November 2014 (Poster W5295).

38 Emmons G, Rowland M. Pharmacokinetic considerations as to when to use dried blood spot sampling. Bioanalysis 2(11), 1791-1796 (2010). 